\title{
Tunable phase-stabilized infrared optical parametric amplifier for high order harmonic generation (Withdrawal Notice)
}

Chunmei Zhang, Pengfei Wei, Yansui Huang, Yuxin Leng, Yinghui Zheng, et al.

Chunmei Zhang, Pengfei Wei, Yansui Huang, Yuxin Leng, Yinghui Zheng, Zhinan Zeng, Ruxin Li, Zhizhan Xu, "Tunable phase-stabilized infrared optical parametric amplifier for high order harmonic generation (Withdrawal Notice)," Proc. SPIE 7430, Laser Beam Shaping X, 743016 (21 August 2009); doi: $10.1117 / 12.831164$

EDIE Event: SPIE Optical Engineering + Applications, 2009, San Diego, California, United States 


\section{Tunable phase-stabilized infrared optical parametric amplifier for high order harmonic generation (withdrawal notice)}

Chunmei Zhang, Pengfei Wei, Yansui Huang, Yuxin Leng, Yinghui Zheng, Ruixin Li and Zhizhan Xu Shanghai Institute of Optics and Fine Mechanics (China)

Proc. SPIE 7430, 743016 (2009)

Online Publication Date: 21 August 2009

Withdrawn from Publication: 22 June 2012

Conference Date: 3-4 August 2009

Conference Location: San Diego, California, United States

Conference Title: Laser Beam Shaping $X$

Conference Chairs: Andrew Forbes, Lizotte Todd

This paper was presented at the SPIE conference indicated above but inadvertently published by SPIE subsequent to its publication in another journal (Optics Letters). The paper has therefore been withdrawn from the SPIE Proceedings by the author and the publisher. The journal paper can be accessed at http://dx.doi.org/10.1364/OL.34.002730. 